\title{
43.
}

\section{ON THE DIFFERENTIAL EQUATIONS WHICH OCCUR IN DYNAMICAL PROBLEMS.}

[From the Cambridge and Dublin Mathematical Journal, vol. II. (1847), pp. 210-219.]

JACOBI, in a very elaborate memoir, "Theoria novi multiplicatoris systemati æquationum differentialium vulgarium applicandi" $\left.{ }^{1}\right)$, has demonstrated a remarkable property of an extensive class of differential equations, namely, that when all the integrals of the system except a single one are known, the remaining integral can always be determined by a quadrature. Included in the class in question are, as Jacobi proceeds to show, the differential equations corresponding to any dynamical problem in which neither the forces nor the equations of condition involve the velocities; i.e. in all ordinary dynamical problems, when all the integrals but one are known, the remaining integral can be determined by quadratures. In the case where the forces and equations of condition are likewise independent of the time, it is immediately seen that the system may be transformed into a system in which the number of equations is less by unity than in the original one, and which does not involve the time, which may afterwards be determined by a quadrature ${ }^{2}$; and, Jacobi's theorem applying to this new system, he arrives at the proposition "In any dynamical problem where the forces and equations of condition contain only the coordinates of the different points of the system, when all the integrals but two are determined, the remaining integrals may be found by quadratures only." In the following paper, which contains the demonstrations of these propositions, the analysis employed by Jacobi has been considerably varied in the details, but the leading features of it are preserved.

${ }_{1}^{1}$ Crelle, t. xxvIr. [1844], pp. 199-268 and t. xxIx. [1845], pp. 213-279 and 333-376. Compare also the memoir in Liouville, t. x. [1845], pp. 337-346.

${ }^{2}$ For, representing the velocities by $x^{\prime}, y^{\prime} \ldots$ the dynamical system takes the form

$$
d t: d x: d y \ldots: d x^{\prime}: d y^{\prime} \ldots=1: x^{\prime}: y^{\prime} \ldots: X: Y \ldots,
$$

and the system in question is simply $d x: d y \ldots: d x^{\prime}: d y^{\prime} \ldots=x^{\prime}: y^{\prime} \ldots: X: Y \ldots$. 
$\S 1$. Let the variables $x, y, z, \ldots$ \&c. be connected with the variables $u, v, w, \ldots$ by the same number of equations, so that the variables of each set may be considered as functions of those of the other set. And assume

$$
d x d y \ldots=\nabla d u d v \ldots ;
$$

if from the functions which equated to zero express the relations between the two sets of variables we form two determinants, the former with the differential caefficients of these functions with respect to $u, v, \ldots$ and the latter with the differential coefficients of the same functions with respect to $x, y, \ldots$ the quotient with its sign changed obtained by dividing the first of these determinants by the second is, as is well known, the value of the function $\nabla$.

\section{Putting for shortness}

and

$$
\begin{aligned}
& \frac{d x}{d u}=\alpha, \quad \frac{d y}{d u}=\beta, \ldots ; \quad \frac{d x}{d v}=\alpha^{\prime}, \frac{d y}{d v}=\beta^{\prime}, \ldots \& \mathrm{c} . \\
& \frac{d u}{d x}=A, \frac{d u}{d y}=B, \ldots ; \quad \frac{d v}{d x}=A^{\prime}, \frac{d v}{d y}=B^{\prime}, \ldots
\end{aligned}
$$

$\nabla$ is the reciprocal of the determinant formed with $A, B, \ldots ; A^{\prime}, B^{\prime}, \ldots$, \&c.; or it is the determinant formed with $\alpha, \beta, \ldots \alpha^{\prime}, \beta^{\prime}, \ldots$, \&c.

From the first of these forms, i.e. considering $\nabla$ as a function of $A, B, \ldots$

$$
\frac{d \nabla}{d A}=-\nabla x, \quad \frac{d \nabla}{d B}=-\nabla \beta, \ldots \quad \frac{d \nabla}{d A^{\prime}}=-\nabla \alpha^{\prime}, \quad \frac{d \nabla}{d B^{\prime}}=-\nabla \beta^{\prime}, \ldots
$$

where the quantities $\alpha, \beta, \ldots \alpha^{\prime}, \beta^{\prime}, \ldots$ and $A, B, \ldots A^{\prime}, B^{\prime}, \ldots$ may be interchanged provided $-\nabla$ be substituted for $\nabla$. (Demonstrations of these formulæ or of some equivalent to them will be found in Jacobi's memoir "De determinantibus functionalibus," Crelle, t. XxII. [(1841) pp. 319-359].)

\section{Hence}

$$
\frac{1}{\nabla} d \nabla+\alpha d A+\beta d B+\ldots+\alpha^{\prime} d A^{\prime}+\beta^{\prime} d B^{\prime}+\ldots=0
$$

or reducing by

$$
\frac{d A}{d y}=\frac{d B}{d x}, \ldots ; \quad \frac{d A^{\prime}}{d y}=\frac{d B^{\prime}}{d x}, \ldots \& c
$$

this becomes

$$
\left.\begin{array}{r}
\frac{1}{\nabla} d \nabla+\alpha\left(\frac{d A}{d x} d x+\frac{d B}{d x} d y+\ldots\right)+\beta\left(\frac{d A}{d y} d x+\frac{d B}{d y} d y+\ldots\right) \ldots \\
+\alpha\left(\frac{d A^{\prime}}{d x} d x+\frac{d B^{\prime}}{d x} d y+\ldots\right)+\beta^{\prime}\left(\frac{d A^{\prime}}{d y} d x+\frac{d B}{d y} d y+\ldots\right) \ldots
\end{array}\right\}=0
$$


or, reducing,

$$
\frac{1}{\nabla} d \nabla+\left(\frac{d A}{d u}+\frac{d A^{\prime}}{d v}+\ldots\right) d x+\left(\frac{d B}{d u}+\frac{d B^{\prime}}{d v}+\ldots\right) d y+\ldots=0
$$

whence separating the differentials and replacing $A, A^{\prime}, \ldots ; B, B^{\prime}, \ldots ;$ by their values

$$
\begin{aligned}
& \frac{1}{\nabla} \frac{d \nabla}{d x}+\frac{d}{d u} \cdot \frac{d u}{d x}+\frac{d}{d v} \cdot \frac{d v}{d x}+\ldots=0 \\
& \frac{1}{\nabla} \frac{d \nabla}{d y}+\frac{d}{d u} \cdot \frac{d u}{d y}+\frac{d}{d v} \cdot \frac{d v}{d y}+\ldots=0
\end{aligned}
$$

(in which $-\nabla, u, v \ldots ; x, y \ldots$ may be substituted for $\nabla, x, y \ldots ; u, v \ldots$ ).

$\S 2$. Let $X, Y \ldots$ be any functions of the variables $x, y, \ldots$ and assume

$$
\begin{aligned}
& U=X \frac{d u}{d x}+Y \frac{d u}{d y}+\ldots \\
& V=X \frac{d v}{d x}+Y \frac{d v}{d y}+\ldots
\end{aligned}
$$

$U, V, \ldots$ being expressed in terms of $u, v, \ldots$. Then

$$
\begin{aligned}
\frac{d U}{d u}+\frac{d V}{d v}+\ldots= & X\left(\frac{d}{d u} \cdot \frac{d u}{d x}+\frac{d}{d v} \cdot \frac{d v}{d x}+\ldots\right)+Y\left(\frac{d}{d u} \cdot \frac{d u}{d y}+\frac{d}{d v} \cdot \frac{d v}{d y}+\ldots\right) \ldots \\
& +\left(\frac{d X}{d u} \cdot \frac{d u}{d x}+\frac{d X}{d v} \cdot \frac{d v}{d x}+\ldots\right)+\left(\frac{d Y}{d u} \cdot \frac{d u}{d y}+\frac{d Y}{d v} \cdot \frac{d v}{d y}+\ldots\right) \ldots
\end{aligned}
$$

i.e.

$$
\nabla\left(\frac{d U}{d u}+\frac{d V}{d v}+\ldots\right)=-\left(X \frac{d \nabla}{d x}+Y \frac{d \nabla}{d y}+\ldots\right)+\nabla\left(\frac{d X}{d x}+\frac{d Y}{d y}+\ldots\right)
$$

Also, whatever be the value of $M$,

$$
U \frac{d M \nabla}{d u}+V \frac{d M \nabla}{d v}+\ldots=X \frac{d M \nabla}{d x}+Y \frac{d M \nabla}{d y}+\ldots
$$

and from these two properties,

$$
\frac{d M \nabla U}{d u}+\frac{d M \nabla V}{d v}+\ldots=\nabla\left(\frac{d M X}{d x}+\frac{d M Y}{d y}+\ldots\right)
$$

$\S 3$. Consider the system of differential equations

$$
d x: d y: d z \ldots=X: Y: Z \ldots
$$

(where, for greater clearness, an additional letter $z$ has been introduced). From these we deduce the equivalent system

$$
d u: d v: d w \ldots=U: V: W \ldots .
$$


Suppose that $u$ and $v$ continue to represent arbitrary functions of $x, y, z, \ldots$ but that the remaining functions $w, \ldots$ are such as to satisfy $W=0, \ldots$ (so that $w, \ldots$ may be considered as the constants introduced by obtaining all the integrals but one of the system of differential equations in $x, y, z, \ldots)$, we have

$$
\frac{d M \nabla U}{d u}+\frac{d M \nabla V}{d v}=\nabla\left(\frac{d M X}{d x}+\frac{d M Y}{d y}+\frac{d M Z}{d z}+\ldots\right) .
$$

Also the only one of the transformed equations which remains to be integrated is

$$
d u: d v=U: V, \quad \text { or } V d u-U d v=0,
$$

(in which it is supposed that $U$ and $V$ are expressed by means of the other integrals in terms of $u$ and $v$ ).

Suppose $M$ can be so determined that

$$
\frac{d M X}{d x}+\frac{d M Y}{d y}+\frac{d M Z}{d z}+\ldots=0
$$

( $M$ is what Jacobi terms the multiplier of the proposed system of differential equations): then

$$
\frac{d M \nabla U}{d u}+\frac{d M \nabla V}{d v}=0
$$

or $M \nabla$ is the multiplier of $V d u-U d v=0$, so that

$$
\int M \nabla(V d u-U d v)=\text { const. }
$$

Hence the theorem:--"Given a multiplier of the system of equations

$$
d x: d y: d z, \ldots=X: Y: Z \ldots
$$

(the meaning of the term being defined as above), then if all the integrals but one of this system are known, the remaining integral depends upon a quadrature."

Jacobi proceeds to discuss a variety of different systems of equations in which it is possible to determine the multiplier $M$. Among the most important of these may be considered the system corresponding to the general problem of Dynamics, which may be discussed under three different forms.

\section{$\S 4$. Lagrange's first form ${ }^{1}$.}

Let the whole series of coordinates, each of them multiplied by the square root of the corresponding mass, be represented by $x, y, \ldots$ and in the same way the whole series of forces, each of them multiplied by the square root of the corresponding mass, by $P, Q, \ldots$; then the equations of motion are

$$
\frac{d^{2} x}{d t^{2}}=X, \quad \frac{d^{2} y}{d t^{2}}=Y, \ldots
$$

${ }^{1}$ I have slightly modified the form so as to avoid the introduction of the masses, and to allow $x$ (for instance) to stand for any one of the coordinates of any of the points, instead of standing for a coordinate parallel to a particular axis. 
where

$$
\begin{aligned}
& X=P+\lambda \frac{d \Theta}{d x}+\mu \frac{d \Phi}{d x}+\ldots \\
& Y=Q+\lambda \frac{d \Theta}{d y}+\mu \frac{d \Phi}{d y}+\ldots
\end{aligned}
$$

where $\Theta=0, \Phi=0, \ldots$ are the equations of condition connecting the variables, and $\lambda, \mu, \ldots$ coefficients to be determined by substituting the values of $\frac{d^{2} x}{d t^{2}}$, \&c. in the equations $\frac{d^{2} \Theta}{d t^{2}}=0, \frac{d^{2} \Phi}{d t^{2}}=0, \&$. It is supposed that as well $P, Q, \ldots$ as $\Theta, \Phi, \ldots$ are independent of the velocities.

In order to reduce these to an analogous form to that previously employed, we have only to write

$$
\frac{d x}{d t}=x^{\prime}, \quad \frac{d y}{d t}=y^{\prime}, \ldots
$$

which gives

$$
\begin{aligned}
d t & : d x: d y: d z \ldots: d x^{\prime}: d y^{\prime}: d z^{\prime} \ldots \\
=1 & : x^{\prime}: y^{\prime}: z^{\prime} \ldots: X: X \quad: \quad: Z \quad \ldots
\end{aligned}
$$

Supposing that $M$ is independent of $x^{\prime}, y^{\prime}, z^{\prime}, \ldots$ the equation on which it depends becomes immediately

$$
\delta M+M\left(\frac{d X}{d x^{\prime}}+\frac{d Y}{d y^{\prime}}+\frac{d Z}{d z^{\prime}}+\ldots\right)=0
$$

where for shortness

$$
\delta=\frac{d}{d t}+x^{\prime} \frac{d}{d x}+y^{\prime} \frac{d}{d y}+z^{\prime} \frac{d}{d z}+\ldots
$$

To reduce this we must first determine the values of $\lambda, \mu \ldots$, and for this we have

$$
\frac{d^{2} \Theta}{d t^{2}}=\delta^{2} \Theta+\frac{d \Theta}{d x} \frac{d^{2} x}{d t^{2}}+\frac{d \Theta}{d y} \frac{d^{2} y}{d t^{2}}+\ldots \quad \quad=0, \& c
$$

i.e.

$$
\begin{aligned}
& \delta^{2} \Theta+P \frac{d \Theta}{d x}+Q \frac{d \Theta}{d y}+\ldots+a \lambda+h \mu+g \nu+\ldots=0 \\
& \delta^{2} \Phi+P \frac{d \Phi}{d x}+Q \frac{d \Phi}{d y}+\ldots+h \lambda+b \mu+f \nu+\ldots=0 \\
& \delta^{2} \Psi+P \frac{d \Psi}{d x}+Q \frac{d \Psi}{d y}+\ldots+g \lambda=f \mu+c \nu+\ldots=0
\end{aligned}
$$


where for greater clearness an additional letter of the series $\Theta, \Phi \ldots$ has been introduced, and where

$$
\begin{aligned}
& a=\left(\frac{d \Theta}{d x}\right)^{2}+\left(\frac{d \Phi}{d y}\right)^{2}+\ldots, \\
& b=\left(\frac{d \Phi}{d x}\right)^{2}+\left(\frac{d \Phi}{d y}\right)^{2}+\ldots, \\
& \vdots \\
& h=\left(\frac{d \Theta}{d x} \frac{d \Phi}{d x}+\frac{d \Theta}{d y} \frac{d \Phi}{d y}\right)+\ldots .
\end{aligned}
$$

Hence differentiating with respect to $x^{\prime}$,

$$
\begin{aligned}
& 2 \delta \frac{d \Theta}{d x}+a \frac{d \lambda}{d x^{\prime}}+h \frac{d \mu}{d x^{\prime}}+g \frac{d \nu}{d x^{\prime}} \ldots=0, \\
& 2 \delta \frac{d \Phi}{d x}+h \frac{d \lambda}{d x^{\prime}}+b \frac{d \mu}{d x^{\prime}}+f \frac{d \nu}{d x^{\prime}} \ldots=0, \\
& y \delta \frac{d \Psi}{d x}+g \frac{d \lambda}{d x^{\prime}}+f \frac{d \mu}{d x^{\prime}}+c \frac{d \nu}{d x^{\prime}} \ldots=0 ;
\end{aligned}
$$

or representing by $K$ the determinant formed with the quantities $a, h, g, \ldots h, b, f, \ldots$ $g, f, c, \ldots$ and by $A, H, G, \ldots H, B, F, \ldots G, F, C, \ldots$ the inverse system of coefficients, we have

$$
\begin{aligned}
& 2\left(A \delta \frac{d \Theta}{d x}+H \delta \frac{d \Phi}{d x}+G \delta \frac{d \Psi}{d x} \ldots\right)+K \frac{d \lambda}{d x^{\prime}}=0 \\
& 2\left(H \delta \frac{d \Theta}{d x}+B \delta \frac{d \Phi}{d x}+F \delta \frac{d \Psi}{d x} \ldots\right)+K \frac{d \mu}{d x^{\prime}}=0 \\
& 2\left(G \delta \frac{d \Theta}{d x}+F \delta \frac{d \Phi}{d x}+C \delta \frac{d \Psi}{d x} \ldots\right)+K \frac{d \nu}{d x^{\prime}}=0
\end{aligned}
$$

whence, multiplying by $\frac{d \Theta}{d x}, \frac{d \Phi}{d x}, \frac{d \Psi}{d x}, \ldots$ and adding,

$$
A \delta\left(\frac{d \Theta}{d x}\right)^{2}+B \delta\left(\frac{d \Phi}{d x}\right)^{2} \ldots+2 H \delta \frac{d \Theta}{d x} \frac{d \Phi}{d x} \ldots+K \frac{d X}{d x^{\prime}}=0,
$$

and, forming the similar equations with the remaining variables and adding,

$$
\begin{gathered}
A \delta a+B \delta b+C \delta c \ldots+2 F \delta f+2 G \delta g+2 H \delta h+\ldots+K\left(\frac{d X}{d x^{\prime}}+\frac{d Y}{d y^{\prime}}+\frac{d Z}{d z^{\prime}} \ldots\right)=0 \\
\delta K+K\left(\frac{d X}{d x^{\prime}}+\frac{d Y}{d y^{\prime}}+\frac{d Z}{d z^{\prime}}+\ldots\right)=0 .
\end{gathered}
$$

C. 
Thus the equation in $M$ reduces itself to

$$
K \delta M-M \delta K=0,
$$

which is satisfied by $M=K$. It may be remarked that. $K$ reduces itself to the sum of the squares of the different functional determinants formed with the differential coefficients of $\Theta, \Phi \ldots$ with respect to the different combinations of as many variables out of the series $x, y \ldots$.

§. 5. Lagrange's second form.

Here the equations of motion are assumed to be

$$
\begin{aligned}
& \frac{d}{d t} \frac{d T}{d x^{\prime}}-\frac{d T}{d x}-P=0 \\
& \frac{d}{d t} \frac{d T}{d y^{\prime}}-\frac{d T}{d y}-Q=0 \\
& \frac{d}{d t} \frac{d T}{d z^{\prime}}-\frac{d T}{d z}-R=0
\end{aligned}
$$

where $2 T$ represents the vis viva of the system, $x, y, z, \ldots$ are the independent variables on which the solution of the problem depends, and $x^{\prime}, y^{\prime}, z^{\prime}, \ldots$ their differential coefficients with respect to the time. It is assumed as before $P, Q, R \ldots$ do not contain $x^{\prime}, y^{\prime}, z^{\prime}, \ldots$

Suppose these equations give

$$
\begin{aligned}
d t: d x: d y: d z \ldots: d x^{\prime}: d y^{\prime}: d z^{\prime} \ldots \\
=1: x^{\prime}: y^{\prime}: z^{\prime} \ldots: X: Y \quad: Z \quad \ldots ;
\end{aligned}
$$

then the equation which determines the multiplier $M$ takes as before the form

$$
\delta M+M\left(\frac{d X}{d x^{\prime}}+\frac{d Y}{d y^{\prime}}+\frac{d Z}{d z^{\prime}}+\ldots\right)=0 .
$$

To reduce this equation, substituting for $T$ its value which is of the form

$$
T=\frac{1}{2}\left(a x^{\prime 2}+b y^{\prime 2}+c z^{\prime 2} \ldots+2 f y^{\prime} z^{\prime}+2 g z^{\prime} x^{\prime}+2 h x^{\prime} y^{\prime} \ldots\right),
$$

and putting for shortness

$$
\begin{aligned}
& L=a x^{\prime}+h y^{\prime}+g z^{\prime} \ldots \\
& M=h x^{\prime}+b y^{\prime}+f z^{\prime} \ldots \\
& N=g x^{\prime}+f y^{\prime}+c z^{\prime} \ldots
\end{aligned}
$$


the equations which determine $X, Y, Z \ldots$ are

$$
\begin{aligned}
& a X+h Y+g Z \ldots+\delta L-\frac{d T}{d x}-P=0 \\
& h X+b Y+f Z \ldots+\delta M-\frac{d T}{d y}-Q=0 \\
& g X+f Y+c Z \ldots+\delta N-\frac{d T}{d z}-R=0
\end{aligned}
$$

Hence, differentiating with respect to $x^{\prime}$,

$$
\begin{aligned}
& a \frac{d X}{d x^{\prime}}+h \frac{d Y}{d x^{\prime}}+g \frac{d Z}{d x^{\prime}} \ldots+\delta a \\
& h \frac{d X}{d x^{\prime}}+b \frac{d Y}{d x^{\prime}}+f \frac{d Z}{d x^{\prime}} \ldots+\delta h+\frac{d M}{d x}-\frac{d L}{d y}=0 \\
& g \frac{d X}{d x^{\prime}}+f \frac{d Y}{d x^{\prime}}+c \frac{d Z}{d x^{\prime}} \ldots+\delta g+\frac{d N}{d x}-\frac{d L}{d z}=0
\end{aligned}
$$

or representing by $K$ the determinant formed with $a, h, g, \ldots h, b, f, \ldots g, f, c, \ldots$ and by $A, H, G, \ldots H, B, F, \ldots G, F, C \ldots$ the inverse system of coefficients, we have

$$
K \frac{d X}{d x^{\prime}}+A \delta a+H \delta h+G \delta g \ldots+\quad * \quad+H\left(\frac{d M}{d x}-\frac{d L}{d y}\right)+G\left(\frac{d N}{d x}-\frac{d L}{d z}\right) \ldots=0,
$$

and similarly

$$
\begin{aligned}
& K \frac{d Y}{d y^{\prime}}+H \delta h+B \delta b+F \delta f \ldots+H\left(\frac{d L}{d y}-\frac{d M}{d x}\right)+\quad+F\left(\frac{d N}{d y}-\frac{d M}{d z}\right) \ldots=0 \\
& K \frac{d Z}{d z^{\prime}}+G \delta g+F \delta f+C \delta c \ldots+G\left(\frac{d L}{d z}-\frac{d N}{d x}\right)+F\left(\frac{d M}{d z}-\frac{d N}{d y}\right)+\quad * \quad \ldots=0
\end{aligned}
$$

Hence, adding,

$$
K\left(\frac{d X}{d x^{\prime}}+\frac{d Y}{d y^{\prime}}+\frac{d Z}{d z^{\prime}} \ldots\right)+A \delta a+B \delta b+C \delta c \ldots+2 F \delta \hat{j}+2 G \delta g+2 H \delta h \ldots=0
$$

and thus we have as before, though with symbols bearing an entirely different signification,

$$
K\left(\frac{d X}{d x^{\prime}}+\frac{d Y}{d y^{\prime}}+\frac{d Z}{d z^{\prime}}+\ldots\right)+\delta K=0 ;
$$

and thence $K \delta M-M \delta K=0$, and $M=K$. 
(The value of $K$ in this section may I think be conveniently termed "the determinant of the vis viva," with respect to the variables $x, y, z, \ldots$. It may be remarked that "the determinant of the vis viva" with respect to any other system of variables $u, v, w, \ldots$ is $=\nabla^{2} K, \nabla$ as before.)

$\S 6$. Third form of the equations of motion. [Hamiltonian form.] Here writing

$$
\frac{d T}{d x^{\prime}}=\xi, \frac{d T}{d y^{\prime}}=\eta, \ldots,
$$

and taking $t, x, y, \ldots \xi, \eta, \ldots$ for the variables of the problem [and considering $T$ to be expressed as a function of these variables: to denote this change it would have been proper to use instead of $T$ a new letter $H$ ] the equations of motion reduce themselves to

$$
\left\{\begin{array}{cc}
\frac{d \xi}{d t}=-\frac{d T}{d x}+P, & \frac{d x}{d t}=\frac{d T}{d \xi} \\
\frac{d \eta}{d t}=-\frac{d T}{d \eta}+Q, & \frac{d y}{d t}=\frac{d T}{d \eta} \\
\vdots & \vdots
\end{array}\right.
$$

or putting for shortness

$$
\begin{cases}P-\frac{d T}{d x}=X, & \frac{d T}{d \xi}=\Xi \\ Q-\frac{d T}{d \eta}=Y, & \frac{d T}{d \eta}=\mathrm{H} \\ \vdots & \vdots\end{cases}
$$

they become

$$
\begin{aligned}
d t: d x: d y: d z \ldots: d \xi: d \eta: d \zeta \ldots \\
=1: \text { 目 }: \mathrm{H}: \Omega \ldots: \bar{X}: Y: Z \ldots .
\end{aligned}
$$

Hence writing the equation in $M$ under the form

$$
\begin{array}{r}
\delta M+M\left(\frac{d \Xi}{d x}+\frac{d \mathrm{H}}{d y}+\ldots+\frac{d X}{d \xi}+\frac{d Y}{d \eta}+\ldots\right)=0 ; \\
\left(\text { where } \delta=\frac{d}{d t}+\Xi \frac{d}{d x}+\mathrm{H} \frac{d}{d y} \ldots+X \frac{d}{d \xi}+Y \frac{d}{d y}+\ldots\right),
\end{array}
$$

we see immediately that $(P, Q \ldots$ being as before independent of the velocities, and consequently of $\xi, \eta, \zeta, \ldots)$,

$$
\frac{d \Xi}{d x}+\frac{d X}{d \xi}=0, \frac{d \mathrm{H}}{d y}+\frac{d Y}{d \eta}=0, \& c .
$$

Hence $\delta M=0$, which is satisfied by $M=1$. 